\title{
Uso y apropiación de herramientas TIC de estudiantes y docentes investigadores en universidades en Medellín*
}

\author{
Use and appropriation of ICT tools of students and researchers \\ professors of universities in Medellin
}

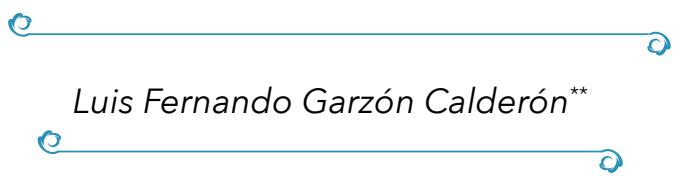

Recibido 12.01. $2018 \bullet$ Arbitrado 22.01. $2018 \bullet$ Aprobado 15.02. 2018

Este artículo es resultado de la investigación intitulada: Uso y apropiación de herramientas TIC en los estudiantes y docentes investigadores en universidades de Medellín, realizada por el Grupo SISCO, Sistemas de Información y Sociedad del Conocimiento, Línea de investigación: Sistemas de Información y Sociedad del Conocimiento, de la Facultad de Ingeniería de la Universidad Católica Luis Amigó de Medellín, en 2015.

* Magister en Administración. Docente - Investigador del Grupo SISCO de la Universidad Católica Luis Amigó, Medellín, Colombia. Correo: luis. garzonca@amigo.edu.co

\section{Resumen}

Este artículo muestra el uso, apropiación e impacto de las herramientas informáticas (TIC) por parte de los investigadores y grupos de investigación de algunas instituciones de educación superior de la ciudad de Medellín, Colombia. Se empleó la metodología cualitativa que permite el análisis de las dinámicas de uso y apropiación de las TIC y su puesta en escena en los procesos de enseñanza-aprendizaje. Igualmente, ofrece los principales resultados de la investigación a la cual hace referencia, tales como: detección de nuevos métodos y herramientas TIC que permiten actualizar, verificar y agilizar la construcción de procesos para la gestión y socialización del conocimiento, y así poder conocer cuál ha sido su aprovechamiento y actualización, para de esta manera ofrecer elementos que permitan direccionar el trabajo investigativo a los investigadores y las instituciones.

Palabras clave: Herramientas informáticas, metodología de la investigación cualitativa, grupos de investigación, tecnologías de la información y la comunicación. 


\section{Abstract}

This article shows the use, appropriation and impact of computer tools (Information and Communication Technology -ICT) by researchers and research groups of institutions of higher education in the city of Medellin, Colombia. It highlights the qualitative methodology that allows the analysis of the dynamics of use and appropriation of the Information and Communication Technology (ICT) and its application in the teaching-learning processes. Likewise, it offers the main results of the research to which it refers, such as: detection of new methods and tools that allow updating, verifying and streamlining the construction of processes for the management and transmission of knowledge, and so we can know what has been its use and updating, in order to offer elements that can direct the investigative work to researchers and institutions.

Keywords: Computer Tools, Research, Research Methodology, Research Groups, Information and Communication Tools.

\section{Introducción}

Los cambios que genera la globalización y la llamada sociedad del conocimiento, exigen una relación con los medios de transformación de datos (software), mediante la eficiencia, eficacia y efectividad de la información, lo que no es ajeno a los grupos de investigación que vienen en un continuo crecimiento en diferentes escenarios (Olivares Carmona, Angulo Armenta, Torres Gastelú y Madrid García, 2016), esto debido a las estrategias desarrolladas por las entidades públicas y privadas, en donde participan universidades, investigadores y, en general, la comunidad de investigación que se refleja en Colciencias. Lo que conduce al desarrollo de proyectos direccionados al fortalecimiento del conocimiento en beneficio de la comunidad (Arévalo Ascanio, Bayona Trillos y Rico Bautista, 2015).

En el país se adelanta un proceso de consolidación de la investigación, muestra de ello se puede ver en el estudio realizado sobre competencias investigativas en los docentes y el uso de las TIC (Paz Saavedra y Fierro Marcillo, 2015), en el cual se involucran tanto los docentes como el uso de las tecnologías y de qué forma aportan en el proceso investigativo dentro de las instituciones (Arcila Calderón, Piñuel Raigada y Calderín Cruz, 2013). Tanto los centros de educación superior como el sector productivo de bienes y servicios están en busca de obtener un mayor nivel de adaptación y apropiación del sistema investigativo entre los estudiantes de postgrado, los egresados, los 
investigadores y los diferentes sectores interesados en el fortalecimiento de la investigación en el país y, de esta forma, lograr potenciar todos los sistemas productivos (Nieto Martin y Rodriguez Conde, 2007).

El crecimiento económico, productivo y social colombiano hace necesario la formulación y ejecución de proyectos de investigación e inversión que fortalezcan el desarrollo del país (Rubiano Arciniégas y Gómez Flórez, 2012), mediante la utilización de herramientas que logren la ejecución de proyectos con el reconocimiento nacional e internacional que vayan de la mano con el crecimiento de la participación del sector académico, investigativo y productivo (Becerra, Zárate Rueda y Rodríguez Quiñónez, 2015). Para lograrlo, se requiere el conocimiento y uso de herramientas informáticas (TIC), que permitan identificar las actualizaciones, la utilización y aplicación en los diferentes sectores productivos e investigativos, permitiendo poner en manos de los investigadores y estudiantes de todos los niveles el desarrollo de nuevas formas de trabajar con los datos y facilitar su análisis para el logro y optimización de la construcción del conocimiento mediante datos ágiles, con alta confiabilidad y una información suministrada oportunamente.

Esta investigación permitió encontrar elementos que sirven como instrumento para las instituciones y personas que tienen sus intereses direccionados al fortalecimiento del proceso investigativo, esperando hacer un aporte importante para el establecimiento de herramientas de apoyo para la construcción del conocimiento indiferente al modelo de investigación utilizado.

Dicho fortalecimiento permite incrementar el número y calidad de los proyectos de investigación, que generan un impacto positivo en la comunidad investigativa, con el propósito de alcanzar una mejora en el desarrollo social y cultural de la población en general (Pérez Pinzón, 2014).

El propósito fundamental del presente estudio se orientó a identificar el uso de las TIC y su impacto en los investigadores, tomando como referente la ciudad de Medellín, lo cual permitió identificar la apropiación de tales herramientas por parte de los investigadores de los grupos de investigación de las universidades: Medellín, EAFIT, Pontificia Bolivariana y Católica Luis Amigó. Otro tanto se hizo con los estudiantes de postgrado de las mismas instituciones de educación superior de la ciudad, mediante el análisis de la información recogida, la cual servirá como soporte a los procesos de investigación de estos, lo que les permite consolidar resultados y nuevas estrategias de trabajo, mediante el uso de las herramientas tecnológicas. 


\section{El desarrollo tecnológico y su papel dentro del contexto}

\section{educativo universitario y su entorno.}

El desarrollo tecnológico asociado a la educación hace que el trabajo con las TIC, dentro de los espacios educativos y de investigación, tenga un papel importante en las universidades y organizaciones (Henao y Cuartas Ramírez, 2016), que deben unir esfuerzos para el fortalecimiento de la educación y la producción mediante la investigación. Esto se logra, mediante el cumplimiento de objetivos sociales y empresariales que apunten al fortalecimiento de la producción y la innovación en el mundo.

La aparición de la tecnología ha hecho que las personas desarrollen nuevas prácticas en las formas de hacer sus trabajos y de direccionar el cumplimiento de sus objetivos (Arbeláez Gómez, 2014), lo cual ha inducido una transición en la sociedad y sus herramientas de trabajo; es decir, ha generado el paso de una sociedad industrial a una de la información (Gandía, 2015).

En la educación han surgido nuevos retos (Fernández Olaskoaga, Fernández Díaz y Gutiérrez Esteban, 2014), se han creado nuevas oportunidades, pero también se han desarrollado nuevas desigualdades; un ejemplo de lo anterior es la llamada brecha digital (Salado Rodríguez, 2011). Por ello, el Banco Mundial definió el acceso que los países tienen a las tecnologías de la información y comunicación, TIC, como uno de los cuatro pilares para medir su grado de avance en el marco de la economía del conocimiento (World Bank Institute, 2008), motivo por el cual se llega a definir la importancia del manejo y uso de la información.

Para la sociología, el creciente interés y avance de las TIC, tiene su origen en el desarrollo tecnológico y en la consecuente construcción de una sociedad de la información y el conocimiento. Allí, los procesos de desarrollo para los diferentes países, se hace de una forma desigual lo cual repercute de una forma directa, en la transformación de cada comunidad según su nivel de utilización y a su influencia en el desarrollo de los diferentes campos educativos y empresariales. Tal intervención permite agilizar y mejorar las herramientas dentro del campo de la investigación e innovación (Tejedor Tejedor, Garcia y Muñoz-Repiso, 2012), mediante una serie de nombres particulares como el de Informatización de la sociedad, sociedad de la comunicación, revolución informacional, informacionalismo, era de la postinformación, sociedad del conocimiento y tercer entorno, como lo señala Crovi Druetta (2002). Todas estas nominaciones, solo señalan que la globalización ha generado un giro en el uso de las herramientas TIC y una 
creciente necesidad relacionada con su uso y apropiación, como factor de disponibilidad para el trabajo de la vida diaria.

La década de los años 80 se caracterizó por ser el momento en el que se inició la revolución de la información; que de hecho, es una revolución del conocimiento y en especial del análisis sistemático y lógico que tiene como base la ciencia cognitiva, más no la electrónica.

Esto se ve claramente en el estudio adelantado por Pérez y Dressler (2006), de la Universidad de Cantabria (España), donde realiza un análisis exploratorio de la relación entre las tecnologías de la información y la comunicación (TIC) y la gestión del conocimiento, llegándose a identificar una serie de herramientas que son de vital importancia para esta investigación, como son: intranets, software de simulación y realidad virtual, workflow, video conferencias, datamining ${ }^{1}$, datawarehouse ${ }^{2}$, inteligencia artificial, motores de búsqueda, gestión documental, mapas de conocimiento y páginas amarillas, mensajería instantánea y correo electrónico, groupware, que son términos que al ser analizados hacen que el mundo de los sistemas de información tome una importancia incalculable al momento de integrarse con el conocimiento y su gestión.

$\mathrm{Al}$ hacer un análisis de las herramientas TIC y de la forma como han evolucionado, otros autores, como (Colas Bravo y De Pablos Pons, 2012), afirman que no cabe la menor duda de que la intervención de las TIC en relación con la cultura científica está contribuyendo de forma acelerada al desarrollo y agilidad en la velocidad y cantidad de información que se puede almacenar, con lo que se contribuye a la investigación, tomando como referente la entrada en funcionamiento de herramientas como la internet y aplicaciones como facebook, twitter, xing, linked-In, instagram y entornos virtuales de enseñanza y aprendizaje que facilitan el acceso a la información y la multiplicación de las actividades que se pueden compartir entre muchos alumnos, docentes e investigadores (Angel Uribe, Vallejo Gómez y Zambrano Acosta, 2015), como se puede apreciar en los centros de educación superior en Morelos México (Torres Velandia, Aguilar Tamayo, Girardo y Villalobos Hernández, 2012), de igual forma se puede identificar el uso dentro de niveles de educación superior fortaleciendo la creación de nuevas formas de crear conocimiento (Ruiz

1 Datamining: (minería de datos), es el conjunto de técnicas y tecnologías que permiten explorar grandes bases de datos, de manera automática o semiautomática, con el objetivo de encontrar patrones repetitivos, tendencias o reglas que expliquen el comportamiento de los datos en un determinado contexto.

2 Datawarehouse: es una base de datos corporativa que se caracteriza por integrar y depurar información de una o más fuentes distintas, para luego procesarla permitiendo su análisis desde infinidad de pespectivas y con grandes velocidades de respuesta. Recuperado de: (http://www.sinnexus.com/business_intelligence/datawarehouse.aspx) Copyright 2007 - 2012 - Sinnexus - Ronda de Outeiro no 116 - 15008 (A Coruña) - Tel/Fax: 881 884859 
Bolívar, 2014), con la ampliación de nuevos espacios y herramientas para la obtención de datos empíricos, al incorporar una cantidad cada vez más amplia y variada de herramientas, donde se incluyen tres tecnologías que son:

- Las redes de comunicación, que facilitan el poder mantener a distancia un intercambio de información y comunicación mezclando voz, imagen y datos.

- Las bases de datos masivas, que ofrecen información organizada a la que se puede acceder mediante información de forma selectiva.

- La computación distribuida, que permite compartir los recursos informáticos mediante redes de computadores.

\section{Las TIC para la transformación del hacer y del actuar en}

\section{una sociedad investigativa}

Uno de los factores fundamentales dentro del trabajo de investigación es fortalecer la forma del cómo lograr en menor tiempo el cumplimiento de objetivos, creando una dinámica de trabajo que permita de forma más eficiente y planificada de hacer las cosas de una mejor manera, donde no solo es importante las herramientas sino cómo es su interacción con las personas que lo desarrollan (Gros, 2016). Se debe tener en cuenta que la comunicación y la interacción social, política, económica, entre otras, hace necesario la utilización de diversos medios (Lotero Echeverri,

2016), ampliando la visión del hombre frente al mundo globalizado mediante la utilización de herramientas informáticas como es el caso de la Internet (Mirete Ruiz, 2016). Las competencias de los orientadores en el ámbito de diagnóstico y desarrollo mediante el uso de las TIC (Tecnologías de la Información y la Comunicación), identifican el uso de Internet como la herramienta que les permite comunicarse con muchos sujetos al mismo tiempo y en espacios distantes. Esto se hace posible para contactar personas que no sería posible con otros medios, debido a motivos de distancia geográfica, idioma, por problemas de movilidad o por cuestiones horarias (Sobrado Fernández, 2006), dejando claro que en las redes de la internet hay un amplio flujo de comunicación y una pluralidad de culturas e idiomas que por otro medio sería imposible identificar para el intercambio de información en el trabajo que se realiza en los procesos investigativos (Pardo Gómez, Izquierdo Lao y Sánchez Ramírez, 2010). De esta forma, se hace necesario interactuar con diversas aplicaciones desarrolladas, especialmente a partir de la denominada web 2.0 (Gamboa Mora y García Sandoval, 2012) y la generalización del video digi- 
tal, creando una nueva cultura investigativa, que cambia sustancialmente las prácticas de investigación (Berzosa Ramos y Arroyo González, 2016), a lo que se añade una serie de dispositivos móviles que han sido probados tanto en la producción de datos, como en el análisis y la representación de ordenadores portátiles PDA (Personal Digital Assistant) asistente digital personal, como son las tablets, smartphones, teléfonos móviles, donde destacan aplicaciones como CAPI (Computer-Assisted Personal Interviewing), que son utilizadas para la ejecución de entrevistas personalizadas asistidas por un ordenador, generando mayor privacidad y movilidad en las entrevistas mediante ayudas informáticas tanto para el entrevistado como para el entrevistador, MCAPI (Mobile Computer-Assisted Personal Interviewing), CAI (Computer-Assisted Interviewing, un conjunto de herramientas de audio asistidas por ordenador que permiten desarrollar auto-entrevistas pregrabadas que pueden contener subtítulos para mayor facilidad en su uso, grabando los resultados mediante una tecnología innovadora con mayor privacidad y estandarización), CATI (Computer-Assisted Telephone Interviewing), ACASI (Audio Computer-Assisted Self-Interviewing), T-ACASI (Telephone AudioComputer-Assisted Self-Interviewing), o CARI (Computer-Assisted Recorded Interviewing), todas orientadas a la recolección de información, que son necesarias para el análisis de la información cualitativa mediante herramientas como son las CAQDAS (Computer-Aided Qualitative Data Analysis), análisis de datos cualitativos asistido por computador, que independientemente de si se utiliza un software específico o no, hacen parte del trabajo desarrollado por el investigador. En este orden de ideas, se toman tres tipos de software para el análisis de datos cualitativos, tomando en primer lugar los recuperadores de texto que hacen la búsqueda de todos los casos de palabras, frases y combinaciones en los cuales se pueden utilizar operadores boléanos que facilitan las búsquedas en uno o más archivos; de igual forma se pueden llevar a cabo funciones propias del análisis de contenido, como la elaboración de listas de palabras, contar palabras, entre otras funciones dentro de las cuales se incluye a los programas gestores de bases textuales como askSam, FolioVIEWS, y MAX, en segundo lugar, se cuenta con un software de codificación y recuperación, que son mucho más útiles para el investigador cualitativo en el momento de dividir el texto en segmentos mediante la asignación de un código (o varios), facilitando las tareas de recuperación, agrupación y ordenación de segmentos y códigos mediante programas como HyperQual, QUALPRO y The Ethnograph, por último, en un tercer lugar, están los programas que surgen en el marco de la Grounded Theory (Teoría Fundamentada) que permiten que el investigador realice de forma más eficiente e incluso con nuevos recursos, todas las tareas que hasta hace poco realizaba de forma manual (fotocopias, tijeras y pegamen- 
to en mano). Estos programas, como AQUAD, ATLASTI, HYPERRESEARCH, NUDITS, MAXQDA o KWALITAN, permiten que los dispositivos móviles estén interconectados en forma inalámbrica para introducir un espacio que contribuya a la construcción de conocimiento en forma global y colaborativa (Caro y Díez, 2005).

En relación con la tecnología móvil los entornos virtuales son utilizados como herramienta para la concepción de movilidad (TIC) (Grané Oró, Crescenzi Lanna y Olmedo Casas, 2013) de igual forma en la enseñanza y aprendizaje, son abordados mediante la integración de la telefonía móvil con el entorno virtual Webinfo, al utilizase en el marco del Programa de Educación a Distancia de la (UNLP) Universidad Nacional de La Plata (Sanz et al., 2006), para quien las TIC juegan un papel fundamental que transforma nuestros modos de hacer y de actuar (Fernández Díaz y Calvo Salvador, 2013), incluyendo las actividades más cotidianas y la forma integral para lograr potencializar nuestras actividades y capacidades, convirtiéndolas en un elemento indispensable para los individuos y sociedades que permiten el desarrollo social y cultural enmarcados en unos procedimientos que transforman el modo de recolección y análisis de la información necesarios para la construcción de herramientas y modelos de investigación. Se destaca la importancia de analizar cómo las TIC en las últimas décadas han logrado un cambio de actitud direccionado a proporcionar una variedad de oportunidades (Jornet, González y García, 2012), destacándose la conformación de redes inteligentes, espontáneas y virtuales, que mediante el intercambio de información generan conocimiento compartido que se nutre alrededor de las tecnologías.

Es importante destacar que existen herramientas informáticas que permiten establecer entre los investigadores una agilidad en la ejecución de múltiples tareas que son significativas dentro de la investigación y la comunicación como es el caso de las redes, equipos portátiles, teléfonos, cámaras, videograbadoras que permiten dar soporte al trabajo de investigación (Gros, 2012). De esta forma se afirma que "Las tecnologías de la información y la comunicación son un componente esencial en la estructuración académico-investigativa virtual en relación a la gestión de la información y uso de conocimientos científicos, técnicos y tecnológicos" (Castillo, 2005, p.35).

La unión de la tecnología con la investigación y su fortalecimiento a través del tiempo y la experiencia hacen parte integral del desarrollo y fortalecimiento de la investigación y la utilización de herramientas informáticas, en este sentido, los aportes de Mruck (2005) pretenden destacar la integración de las múltiples herramientas en línea y fuera de ella, y se refiere a tres tipos de servicios: a) el apoyo para archivar datos primarios y el acceso a bases de 
datos para el análisis de datos cualitativos; b) la consultoría en asuntos sobre enseñanza e investigación cualitativa y c) las bases de información, comunicación, publicación y posibilidades para distribuir, manejar y compartir conocimiento, Mruck sugiere, además, estar vinculados a redes que comparten, coordinan y generan servicios disponibles para la construcción de recursos valiosos en el desarrollo de trabajos realizados por investigadores a través del mundo, y así fortalecer los bancos de datos existentes como soporte para futuras investigaciones.

\section{El investigador frente al desarrollo e innovación}

\section{tecnológica}

Con los grupos colombianos de investigación clasificados en A y A1 por Colciencias, se rastrearon las herramientas tecnológicas y su uso en los procesos de investigación por parte de estos. De análoga forma, se procedió con los investigadores y estudiantes de maestría y doctorado de la ciudad de Medellín, según los objetivos planteados en la investigación: determinar las herramientas informáticas (TIC) que intervienen en la investigación, la metodología de la investigación y el uso que se les da en los centros de educación superior de la ciudad de Medellín, partiendo de los desafíos de un mundo globalizado en una sociedad que está cambiando aceleradamente, donde los investigadores y grupos de investigación deben impulsar el fortalecimiento de la investigación mediante el uso de todas las herramientas existentes y el conocimiento sobre su aplicación (Carvajal Torres y De la Torre, 2016), con transformaciones que favorezcan a todos los sectores de la comunidad, dando soporte no sólo a las empresas para el mejoramiento de la producción, sino para fortalecer el crecimiento económico y cultural del país y el entorno mundial.

Dentro de las herramientas más relevantes que las TIC han puesto al servicio de la investigación, sin lugar a dudas están las bases de datos científicas, que manejan información relacionada con artículos científicos, revistas, libros, ponencias y reportes de investigación, tesis y demás trabajos de investigación relevantes que pueden ser consultados desde las universidades por investigadores y estudiantes vinculados por los centros de educación.

Cada vez son más útiles los sistemas de mensajería instantánea como el correo electrónico, los chat y video conferencias que posibilitan la comunicación al momento de realizar contacto con cualquier persona del planeta para intercambiar ideas y compartir criterios y experiencias. El mejoramiento de software de búsqueda, se soporta en los motores de bases de datos, la recolección 
y manipulación de datos utilizados en la minería de datos (Caro, Fuentes y Soto, 2013) se basa en el alineamiento de almacenes de datos y sugiere que es necesario garantizar que los almacenes de datos estén alineados a los objetivos que se esperan alcanzar, unidos a la estrategia que permite el alcance del éxito, para los equipos de proyectos el almacén de datos se debe tomar como una herramienta estratégica, de esta forma los datos y la información pueden ser utilizados por los investigadores con el fin de analizar el desarrollo estratégico de la investigación, mediante los datos tomados desde puntos estratégicos como las entidades bancarias o almacenes de cadena que pueden tomar la información de los datos suministrados por los clientes en tiempo real desde los movimientos financieros, las tarjetas de crédito, el material multimedia y tutoriales interactivos que se convierten en soporte para enriquecer las experiencias de investigadores y grupos de investigación que son vitales para el desarrollo y fortalecimiento del trabajo investigativo.

Lo anterior permite visualizar las herramientas informáticas, el procesamiento de la documentación digital, las herramientas de búsqueda, la revisión y comunicación, como parte fundamental para el fortalecimiento de la investigación de tal forma que lo tecnológico sustente a las TIC y lo investigativo en los contenidos científicos y académicos, entregue soporte a la academia, la empresa y la investigación desde la innovación, la construcción colaborativa del contenido científico y la creación de conocimientos como parte fundamental en la elaboración de nuevos modelos .

El valor que toma el software en los proyectos de investigación es prioritario por su innovación y agilidad en el procesamiento de los datos, lo que no puede generar una falsa sensación de agilidad y seguridad en la finalización de los procesos que se desarrollan en los proyectos de investigación, pues es necesario tener en cuenta que hay muchas cosas que los programas del computador no saben hacer, ni pueden analizar, lo que conlleva a afirmar que es más valioso el conocimiento sobre la gestión del proyecto, que la herramienta de software a utilizar, quedando claro que el investigador o grupo de investigadores son quienes determinan la dirección del proyecto con las decisiones que se toman, especificando en qué formatos y cuántos datos o información se debe procesar y qué herramientas utilizar (Sánchez Robayo y Torres Duarte, 2016); por otro lado, son ellos quienes tienen la responsabilidad del proyecto y la verificación de los datos, tomando como base que una mala información suministrada genera una evaluación y resultados erróneos. Por último, debe tenerse en cuenta que el trabajo que se desarrolla involucra el bienestar del ser humano que enfrenta los problemas y soluciones que deben resolverse (Heerkens, 2002). 
De acuerdo con Crovi Druetta (2002), la clave para mantener el liderazgo en la economía y la tecnología, estará en la posición social que tengan los profesionales del conocimiento y la aceptación social de sus valores. Es importante enfatizar que la calidad de los resultados depende directamente de la posibilidad de medición, lo que implica la recolección de los datos $\mathrm{y}$ su procesamiento dejando claro la importancia de que las herramientas informáticas sean visualizadas no como un obstáculo sino como una ayuda en el proceso investigativo (Torres Velandia y Jaimes Cruz, 2015), e identifica la necesidad de utilizar herramientas que permitan al investigador o grupo de investigación efectuar los cambios necesarios en el momento de hacer las consultas requeridas con fácil acceso a la información y seguridad que se requiere, mejorando el tiempo en que se obtienen los datos, para establecer la agilidad en el desarrollo de los pasos necesarios para la ejecución de proyectos direccionados a la investigación.

Esta investigación también ha permitido observar la importancia que tiene para el investigador la apropiación de las herramientas tecnológicas y su uso, frente a los desarrollos e innovaciones, como sujeto fundamental por su capacidad de adaptabilidad para la recolección de los datos (Guacaneme, Zambrano y Gómez, 2016), destacando su capacidad holística en un contexto global, donde cobra gran relevancia la agilidad en las bases de datos y su interpretación de respuestas a través de la intuición, los sentimientos, las sensaciones, entre otros, y la utilización de técnicas como entrevistas, observaciones, relatos, discursos, narraciones, historias de vida, etc. (Colas Bravo y De Pablos Pons, 2012), utilizan diversas herramientas tecnológicas como las redes sociales para localizar colectivos específicos y que constituyen, según los autores, poblaciones ocultas o invisibles socialmente; además de otros colectivos marginales tales como delincuentes, drogadictos, inmigrantes, etc., cuya localización y acceso son problemáticos para obtener información y triangular los resultados obtenidos.

\section{Las herramientas informáticas como factor de producción y}

\section{divulgación en la investigación.}

El conocimiento y uso de herramientas informáticas en estudiantes y docentes permite la integración y fortalecimiento de la educación en investigación mediante el uso y apropiación de conocimientos (Escofet Roig, López y Álvarez, 2014) permitiendo que la investigación y la metodología de la investigación dentro de las universidades (Capanegra, Cabrera, Aguilar y Jorda, 2016) en conjunto con las empresas avancen con mayor velocidad y agilidad 
en los resultados obtenidos (Isaza Domínguez, Vargas Guativa y Preciado, 2016), para ser rápidamente divulgados y con mayor exactitud. Los retos planteados por las TIC al proceso de investigación científica son relativos a la gestión y procesamiento de la información, a la comunicación tanto sincrónica como asincrónica, en el cambio de rol de profesores, estudiantes y otros sujetos que se manifiestan, en la utilización de estrategias dinámicas participativas y colaborativas, en la realización de proyectos conjuntos que trasciendan el marco institucional, en el tránsito del trabajo aislado al desarrollo de tareas en conjunto (Nieto Martin y Rodriguez Conde, 2007), con la formación de comunidades científicas en temáticas comunes y redes de investigación y equipos de trabajo (Pardo Gómez, Izquierdo-Lao y Sánchez Ramírez, 2010), lo que permite asegurar la intervención tanto del software como del hardware y su evolución para el fortalecimiento de las herramientas que dan soporte al trabajo de investigación.

Son muchas las ventajas y aportes que las tecnologías digitales pueden realizar en el trabajo conjunto de investigadores en la actualidad y a todo nivel (Alaís Grillo, Leguizamón Sotto y Sarmiento Ceballos, 2015; Navarrete Calao y Vargas Arteaga, 2015); no obstante, aún en algunos ámbitos del quehacer humano (Ramírez-de-La-Piscina-Martínez, 2013), la utilización de las TIC parece generar, cuando menos, controversia y un excesivo grado de suspicacia por lo que resulta obvio que la utilización de estos medios pueden tener tanto ventajas como inconvenientes (Martínez Flores y Torres Barzabal, 2017).

De otro lado, Colas Bravo y De Pablos Pons (2012), al referirse a la selección muestral de carácter cualitativo, señalaron ciertos aspectos que son determinantes en la investigación y la metodología de la investigación: la identificación de la muestra, la localización de la muestra y la información que aporta la misma, que toman valor significativo en el momento del análisis de la información y la cual requiere para la toma de la muestra contar con herramientas que podemos identificar cómo son las redes sociales, tales como Facebook, Twitter, Xing, Linked-In, agrupando colectivos que comparten intereses, costumbres y modelos de consumo que permite aglutinar comunidades que tienen rasgos similares tomadas como fuentes para identificar muestras de interés para los investigadores, grupos de investigación y el medio con el que comparten las experiencias.

Finalmente, se puede decir que las TIC se presentan como potentes herramientas con la capacidad para mejorar considerablemente la calidad de la investigación cualitativa y cuantitativa, teniendo impacto en todos los criterios de calidad tradicionalmente utilizados en la investigación como son: credibilidad, transferibilidad, dependencia y confiabilidad. Las TIC facilitan y mejoran 
los procedimientos que se aplican en la metodología de la investigación para asegurar su agilidad en el manejo de los datos ayudando con la calidad científica, lo que se puede visualizar en procesos como son la recogida mecánica de abundantes datos, contrastación de los resultados con los participantes, triangulación (de fuentes, metodológicas, informantes, etc.), el muestreo teórico, y las observaciones persistentes o dilatadas en el tiempo.

\section{Metodología}

Inicialmente se realizó una búsqueda exploratoria direccionada a encontrar el objeto de investigación y de allí determinar con precisión el problema de investigación, pues referirse al uso de las TIC en la investigación y la metodología de la investigación resultaba un asunto demasiado amplio y complejo.

Para definir el problema de investigación, se partió de una amplia búsqueda de documentos y artículos científicos, que sirvieron de soporte para hacer un análisis de la investigación y establecer un direccionamiento de la información. Sin embargo, el aporte más significativo, en relación con esta búsqueda de precisión del problema de investigación, se encontró en las entrevistas con expertos.

Inicialmente se realizaron entrevistas con Ingenieros de Sistemas expertos en herramientas para la de recolección de datos, con quienes se buscó identificar la complejidad del tema, las herramientas de uso, el alcance y alto nivel de profundización requerido que debía dársele a la investigación. Lo anterior, con el propósito de establecer la importancia en el uso de herramientas informáticas que permiten a los investigadores realizar su trabajo de forma analítica, productiva y con una comunicación globalizada, sin importar el espacio geográfico, así como también lograr almacenar grandes cantidades de información en espacios virtuales.

Posterior a ello, se realizaron entrevistas a expertos en investigación de la Universidad Católica Luis Amigó, lo que permitió definir el ¿Por qué? o ¿Para qué? de la investigación, dando la posibilidad de identificar con claridad la necesidad de utilizar un enfoque cualitativo en la investigación, "se debe identificar si se van a usar las herramientas informáticas, en qué forma y bajo qué enfoque, si van a ser utilizadas en un proceso de análisis cuantitativo o cualitativo. El otro tema, es el referido al manejo de resultados o de la misma gestión de conocimiento con el software a aplicar, si permiten representación visual de conocimiento, o si son herramientas de gestión o de cálculo de datos o información" (entrevista expertos, 26/06/2014). 
De igual manera, se identificó la población y muestra del estudio tomando como base los GrupLac de Colciencias y un grupo de universidades de Medellín para dar inicio a la construcción de la investigación y de los instrumentos necesarios para desarrollar las encuestas y entrevistas, esto ayudó a aclarar cómo elaborar las preguntas y qué términos utilizar para identificar un lenguaje fácil de entender por las personas encuestadas y entrevistadas para la recolección de la información "[...] el usuario puede tener un concepto muy diferente al que nosotros tenemos o estamos entendiendo por TIC" (entrevista expertos, 26/06/2014), todo lo anterior fue revisado y avalado por los expertos.

Una vez recolectada la información se tabuló y fue analizada con un grupo de expertos de la universidad Luis Amigó, lo que permitió identificar un grupo de herramientas informáticas (TIC), su uso, apropiación e impacto destacando el enfoque a donde están direccionadas, tomando como referente los investigadores, grupos de investigación e instituciones.

Mediante la exposición de resultados se logró identificar las características y herramientas utilizadas en la construcción de la investigación tanto cualitativa como cuantitativa, lo que generó una valiosa visualización para la generación de nuevos modelos de recolección y organización de datos e información, planeación y desarrollo de modelos de análisis de información y uso de herramientas poco conocidas dentro del contexto de la investigación. Lo anterior permite a las instituciones evidenciar la importancia de la innovación y de la capacitación de los investigadores y estudiantes, impactando en la mejora continua del trabajo investigativo y dando cumplimiento a los objetivos planteados.

\section{Resultados y discusión}

El sondeo se realizó con grupos de investigación registrados a junio de 2013 en los GrupLac de Colciencias. A partir de la información allí registrada, se estableció contacto con los investigadores de los grupos de investigación, mediante correo electrónico y se envió un formulario a 330 investigadores en Colombia, de los cuales respondieron 69. Al tiempo se hicieron entrevistas con expertos de las universidades de Medellín, EAFIT, Pontificia Bolivariana y Católica Luis Amigó lo que permitió identificar las siguientes categorías:

\section{Experiencia en el uso de las TIC.}

Sobre el uso de las herramientas informáticas por parte de los investigadores se evidencia, de manera representativa, que el $76 \%$ de la población 
encuestada tiene acceso a herramientas informáticas, el 60\% de ellos las utilizan y el 50\% reconoce el impacto que la tecnología tiene en los procesos de investigación, sin embargo, sólo el 40\% mencionan tener conocimiento específico sobre ellas, lo cual se convierte en una oportunidad para que se sigan formando y cualifiquen su conocimiento sobre dichas herramientas.

Al preguntar a los profesores y estudiantes de maestrías y doctorados acerca del papel que desempeñan las TIC en el trabajo de investigación, algunos responden a partir de la experiencia que han tenido en la búsqueda de información: "Uno encuentra mucho documento fundamental en sitios web internacionales, por ejemplo la Universidad de la Rioja, en España, tiene un sitio que se llama Tialnet.com con revistas indexadas de un alto peso en materia jurídica y social; la Universidad Autónoma de México tiene en la página web abundante material bibliográfico para estos temas, que en internet es fundamental" (Estudiante doctorado en Derecho, 26/07/2014). También se encontró un variado número de formas de identificar las herramientas informáticas y su efectividad de uso, como puede apreciarse en la respuesta de un estudiante de doctorado en Derecho: "Yo creo que con lo que existe actualmente podría hacerse un trabajo investigativo, yo creo que nosotros ya por la edad somos más cavernarios, que los muchachos de ahora que nacen sistematizados; yo creo que nosotros somos más ratones de biblioteca; creo que ese trabajo es más de uno, es más humano, que de una máquina; yo creo más en la capacidad de uno de inferir en las conclusiones, que en un trabajo que pueda hacer un computador" (Estudiante doctorado de Derecho, 26/07/2014).

El conocimiento y uso que se tiene de las herramientas informáticas depende mucho del área a la que pertenecen los investigadores, ejemplo de ello son las personas que laboran en el área del Derecho, quienes a la pregunta que indaga si el acceso a las herramientas TIC le facilitan el trabajo, mencionaron que solo hacen uso de herramientas para la búsqueda de información en bases de datos y almacenamiento de información a partir de procesadores de texto, a la vez que manifestaron su desconocimiento de las demás herramientas informáticas.

De otro lado al indagar a un estudiante de Doctorado en Administración con relación al conocimiento, uso de las herramientas informáticas y las dificultades que se presentan principalmente para cierto tipo de investigadores relata que: "Lo principal a tener en cuenta es que yo no soy hija de las TIC, cierto, a pesar de que yo tengo 42 años, pues soy una persona joven pero no soy hija de las TIC como lo puede ser mi hija, cierto, porque tiene 9 años, porque para ella todas estas cuestiones se vuelven más familiares; o sea: yo trabajé, podría decir hasta la maestría, mucho a mano y escribiendo, ya poco a poco me fui 
metiendo, pues algo tarde, en algo tan simple como es el uso del computador y eso me tocó aprenderlo, el uso del office es una dificultad; hay veces ese tipo de cosas le cuesta a uno" (Estudiante Doctorado en Administración, 26/06/2014).

Se logró identificar que el uso y manejo de las herramientas informáticas depende en gran parte del tipo de formación del investigador y sus necesidades de uso en su trabajo cotidiano.

En relación con los niveles de conocimiento y de uso de los conceptos básicos asociados a las TIC (conexión a internet, ADSL, velocidad de acceso, ancho de banda, aplicaciones, sistema operativo), las respuestas de los entrevistados son un tanto contradictorias, pues aunque manifiestan un bajo conocimiento de los conceptos también señalan un alto uso, lo que permite ver en las herramientas TIC un recurso amigable que requiere ser incluido dentro de las técnicas utilizadas en la investigación y de las cuales se debe tener conocimiento no solo de su uso, sino sobre las bondades y la forma de hacer su adquisición como recursos TIC para la investigación, permitiendo estimular la participación del grupo de trabajo, el investigador y la institución.

Se puede identificar una interpretación clara sobre la necesidad que tienen los estudiantes, investigadores y entidades que están vinculadas con la investigación, de fortalecer el nivel de conocimiento de las herramientas informáticas, no solo sobre su uso, sino también sobre las bondades que se pueden encontrar en ellas, mediante el fortalecimiento de políticas, planes y estrategias orientadas a la construcción de un modelo institucional que permita que las personas en forma gradual fortalezcan el uso y conocimiento de las TIC y su aplicación en las actividades de trabajo.

\section{Motivación hacia el uso de las TIC.}

Otros estudiantes de postgrados, los docentes y asesores de trabajos de grado y tesis doctorales permiten identificar claramente el interés en las herramientas informáticas: "Creo que si las personas conocen las herramientas pueden hacer un mejor uso de ellas. Nosotros hemos visto en los programas de maestría de Derecho procesal, que muchas personas no conocen las herramientas para la investigación, como bases de datos, ya que es un término elevado para ellos, lo que hace que desaprovechen esa oportunidad, puesto que ahí es donde se puede encontrar el material más importante, sobre las que se pueden soportar las investigaciones. Muchas personas hacen investigación buscando en google y creo que eso es un error" (Profesor de Maestría y Doctorado Derecho, 11/07/2014). 


\section{Colaboración entre grupos de investigación y sus formas de}

\section{comunicación.}

Al preguntar a los investigadores sobre el uso que le dan a las herramientas informáticas más utilizadas para comunicarse, el 96\% de la población encuestada utiliza el correo electronico y en la misma proporción utilizan los foros; el $87 \%$ usa la mensajeria instantanea /chat, lo cual permite evidenciar un alto uso e impacto de la comunicación, mediante la implementación de las TIC en sus procesos investigativos.

En la investigación es clave la comunicación e intercambio de experiencias con otros colegas investigadores al igual que para la generación de espacios muéstrales. Tanto para los estudiantes como para los profesores e investigadores, la comunicación es clave para la construcción de problemas de investigación, lo que depende de forma directa del uso de herramientas informáticas, y que a medida que son mejoradas facilitan el trabajo investigativo: "Nosotros pertenecemos a una red, es la red interuniversitaria de desarrollo procesal, allí es donde están los demás investigadores, compartimos información. La verdad esta red tiene espacio virtual, en un principio no se estaba utilizando porque muchas personas seguimos pegados al correo electrónico o a la llamada por el celular, de esa manera nos comunicamos, pero no se utiliza ninguna otra herramienta más" (Profesor Maestría y Doctorado Derecho, 11/07/2014).

En este sentido la gran mayoría de las universidades han buscado implementar y desarrollar áreas de TIC que sirvan para la investigación y la docencia. Se han podido identificar herramientas que son de uso significativo en el trabajo de investigación, como es el caso de las utilizadas por la Universidad EAFIT a través del Proyecto 50, con las que pueden interactuar no solo los estudiante y profesores de la Universidad, sino todas las personas vinculadas a proyectos de investigación y que hacen parte de múltiples grupos de investigación en el país y fuera de él, dejando claro la fortaleza de las herramientas informáticas y su forma de interactuar en el trabajo de investigación.

Los estudiantes de doctorado se apoyan en estas herramientas de manera significativa, uno de ellos mencionó: "Ya en la experiencia que tuvimos nosotros en ese planteamiento inicial de la investigación que nos reunimos varios profesores de varias universidades de Colombia desarrollamos otra cosa más, como actividad de la que hacíamos mucho uso que eran los foros virtuales, a través de la plataforma que nosotros tenemos de EAFIT interactiva, que es como la que más nos facilita el uso de esas herramientas hasta ahora, por 
ejemplo, con herramientas de Proyecto 50 que es el área de nosotros especializada en TIC, no he interactuado mucho todavía pero creo que lo voy a tener que hacer; estamos en este momento con algo, que pienso, podré utilizar para armar todo el marco teórico de mi trabajo de investigación; estamos incursionando por allá en un curso en el que soy coordinadora, en el diseño de itinerario de aprendizaje. Ese concepto ayudado por las herramientas que tiene Proyecto 50, me puede servir mucho para diseñar un esquema general de la que va a ser el marco teórico de mi investigación" (Estudiante Doctorado Administración 21/06/2014).

Es importante resaltar el uso de otras herramientas para la comunicación entre los investigadores, quienes dicen conocer google docs, mendeley, share point, páginas web, team viewer, yahoo group y google group, se parte del uso de un correo instantáneo y colaborativo donde desaparecen las fronteras y permite a los investigadores intercambiar opiniones y conocimiento, al igual que hacer contacto con espacios muéstrales a los que de otra forma seria imposible llegar. De esta manera se genera un espacio de trabajo globalizado direccionado al crecimiento del conocimiento y la información.

\section{Apoyo para la publicación y divulgación de resultados}

Al preguntar a los investigadores sobre el uso y conocimiento de las herramientas de búsqueda que los investigadores de los grupos de Colciencias utilizan, se encuentra que los motores de búsqueda (buscadores) son utilizados por un $95 \%$ de ellos, mientras que las bases de datos científicas y académicas en un $91 \%$ y el campus virtual de su universidad un $80 \%$. Por otro lado, en relación a las herramientas informáticas utilizadas por los investigadores en la organización de las referencias bibliográficas, se identifica que los más usados son EndNote con un 37\% y Zotero con un 20\%.

Los investigadores señalan, que el uso de herramientas para la comunicación, búsqueda de información y organización de las referencias bibliográficas, se hace de forma equitativa, que se reparte en desarrollos propios y revistas que se encuentran en las bases de datos nacionales e internacionales.

Lo que se puede afianzar en opiniones como: "Desde que el mundo se abre a partir de la conectividad en estructuras de redes, pienso que el trabajo investigativo se enriquece, en la medida en que los investigadores se conecten a través de redes para permitir su movilidad, a través del uso de herramientas tecnológicas que se convierten en soporte fundamental para la conectividad. Razón por la cual he podido publicar trabajos por fuera del país con otros 
profesores y he podido publicar en revistas importantes, y esto no se hubiera logrado sin la intermediación de estas herramientas" (Profesor Maestría en Administración, 05/09/2014).

\section{Limitaciones más significativas para la utilización de las}

\section{herramientas informáticas en el trabajo de investigación.}

En relación con las limitaciones más significativas para la utilización de las herramientas informáticas en el trabajo de investigación, los investigadores precisan que no cuentan con los equipos requeridos, tampoco se cuenta con suficiente formación/capacitación por lo cual es necesario desarrollar programas o aplicaciones para agilizar el trabajo de investigación.

El conjunto de limitaciones permite identificar la necesidad que tienen las entidades de generar planes y establecer políticas, que brinden a los estudiantes de maestría y doctorado, docentes e investigadores, el soporte necesario para identificar, las herramientas requeridas para el manejo de datos e información en el trabajo investigativo acorde con el enfoque cualitativo o cuantitativo utilizado y de esta manera generar agilidad, veracidad, oportunidad y calidad en el análisis de la información, obteniendo así la optimización de la producción investigativa.

\section{Finalidad de uso de las TIC}

$\mathrm{Al}$ indagar sobre las herramientas informáticas más utilizadas por los investigadores para la recolección de la información y datos, se evidencia que los más usados son Evernote con un 33\% y Ubemote con un $12 \%$.

En relación con las herramientas informáticas más utilizadas por los investigadores para el análisis y organización de la información, los resultados muestran que los más representativos son Word con un $92 \%$ y Excel con un $84 \%$. Por otro lado, las herramientas más utilizadas para la elaboración del informe final de investigación son Word con un 96\%, Excel con un 83\%, PowerPoint con un 91\% y Prezzi 62\%.

La información anterior evidencia un marcado uso de las herramientas tradicionales como Word, Excel, Power Point y Prezzi, pero no de forma eficiente, por el desconocimiento de todas las bondades que ellas pueden generar, lo anterior puede significar que no hay una divulgación sobre el uso y ventajas de la utilización de estas herramientas y otras que están entrando al mercado 
de forma permanente al igual que sus actualizaciones, generando la necesidad en las instituciones y en los investigadores, de fomentar la mayor participación, en encuentros de investigación nacional e internacional que les permitan una actualización permanente acorde con el trabajo de investigación.

Las herramientas informáticas también pueden presentar dificultades en muchos otros sentidos como se puede entender en el siguiente planteamiento: "Yo he usado principalmente para mi tesis de doctorado las bases de datos y la plataforma de Colciencias, para identificar qué se está haciendo desde los grupos y lo que han hecho en los últimos 10 años en los temas de ambiente y de agua, y me he dado cuenta que el enfoque es muy técnico, no son enfoques que me interesen, pero me interesa saber para tener un panorama de los que hay en Colombia, en los grupos de investigación; y ya a nivel internacional he mirado ISIS y SCOPUS, como bases de datos muy reconocidas en el mundo sobre autores que han trabajado o están trabajando el tema, y en los últimos 10 años no son muchos los artículos que hay, es muy poco lo que se ha investigado, por lo que el tema es muy nuevo y hay mucho que profundizar." (Estudiante doctorado Administración, 26/06/2014).

En relación con la información y la cantidad de resultados que pueden generarse de la investigación, un aspecto clave hoy en un mundo global, es la velocidad con que las herramientas informáticas hacen posible almacenar y agrupar grandes cantidades de datos, los cuales deben ser procesados por herramientas estadísticas para agilizar el trabajo al investigador, lo que garantiza el uso eficiente de la información procesada frente a la cantidad evaluada para fortalecer el trabajo de investigación, como se puede entender en lo expuesto en la siguiente entrevista "El trabajo de investigación pienso que es un problema de carácter humano y es la capacidad de procesar la información; es decir, gracias a las TIC tenemos mayor cantidad de información, pero precisamente eso que es tan bondadoso, se convierte en un problema. Las preguntas son: ¿cómo gestiono la información?, ¿cómo la proceso?; yo creo que ahí es donde se encuentra la principal debilidad, termina uno volviéndose muy selectivo o sin tener criterios de fondo para hacerlo o carece uno de capacidades técnicas para el procesamiento de la información de tipo cuantitativo" (Profesor Maestría Administración, 05/09/2014).

El manejo de otros idiomas se hace fundamental en la construcción de nuevo conocimiento para lo que se pueden utilizar herramientas informáticas de traducción, de igual forma el manejo en redes de documentos en distintos idiomas funciona como ayuda integral del trabajo de investigación. En este sentido, es relevante tener en cuenta lo expuesto por un profesor de maestría y doctorado. "A mi si me parece muy importante la investigación que están 
haciendo ustedes, y creo que les va a traer muchos resultados en aspectos que los investigadores deben mejorar; y es precisamente en el manejo de las herramientas. Creo que muchas personas no utilizan esas herramientas tecnológicas quizás porque no las conocen, no les han dado capacitación o las consideran demasiado engorrosas; pero al fin de cuentas, uno pierde más tiempo cuando no utiliza las herramientas. Un aspecto que se debería tener en cuenta es lo relacionado con la formación de docentes en otros idiomas, en mi caso particular se me facilita porque todas vienen en inglés y si uno no tiene esa formación, no puede comprender algunos textos y hacer el uso adecuado de todas esas herramientas" (Profesor Maestría y Doctorado Derecho, 11/07/2014).

Los investigadores dejan claro que no puede olvidarse la importancia del uso de los documentos físicos que se encuentran en las bibliotecas, pues al analizar la frecuencia en el uso de libros, documentos y otros materiales para la consulta de temas de investigación, los investigadores responden que lo utilizan: alguna vez el $46 \%$, muchas veces, el $21 \%$ y bastantes veces, el $33 \%$. Lo anterior permite identificar cómo el uso y conocimiento de las tecnologías, no es ajeno al común de las personas, pero si denota el grado de importancia que tiene el identificar las herramientas informáticas, su utilización y sobre todo el saber acerca de las bondades que estas brindan sin desconocer el uso del material físico. De esta manera, se entra en un contexto donde se trabaja la información desde el uso y apropiación de herramientas que construyen con la investigación mediante espacios propicios, proporcionando al investigador el ahorro de tiempo en la recolección, análisis y generación de informes, que disminuyen el costo en la construcción de nuevo conocimiento.

\section{Conclusiones}

Los resultados obtenidos están estrechamente relacionados con el objetivo de la investigación y pueden derivar en futuras investigaciones sobre cómo las TIC impactan en los resultados finales de las investigaciones, además de identificar el potencial que tienen las universidades en el diseño de programas o software que permitan fortalecer el trabajo de investigación mediante la utilización de herramientas informáticas como puede verse en la entrevista con el grupo de investigación EAV- UPB (Educación en Ambientes Virtuales, de la Universidad Pontificia Bolivariana), quienes con una experiencia de más de diez años han identificado un esquema de trabajo en relación con las TIC, lo que deja ver la necesidad de realizar capacitaciones sobre software y hardware de procesamiento de datos, según áreas específicas del conocimiento. 
El sondeo realizado a los grupos de investigación, puede ayudar a los mismos a desarrollar estrategias y a orientar los avances y resultados con más precisión y con un mejor manejo de flujo de la información, lo que se convierte en un aporte valioso de las herramientas tecnológicas, permitiendo la transcripción y análisis de los datos que surgen en la investigación, en este sentido, se puede evidenciar a las TIC como potentes instrumentos para optimizar los tiempos y cantidad de información recolectada. Lo anterior deja clara la importancia de dar a conocer las bondades y alcances de las herramientas TIC dentro de la comunidad investigativa, tomándolo como una estrategia de mejora continua.

Las TIC facilitan y mejoran los procedimientos investigativos e identifican oportunidades académicas (cursos, estudios, conferencias) que posibilitan múltiples usos en la docencia como se puede visualizar en las entrevistas realizadas a los expertos, quienes mostraron interés en el grado de apropiación y uso de las herramientas informáticas a las que tenían acceso en las universidades para desarrollar los proyectos de investigación; y además, que fomentan la comunicación entre los grupos de investigación, garantizando que los diferentes miembros puedan participar a través del uso de estas indiferente del lugar donde se encuentren ubicados.

Algunas de las herramientas más utilizadas son las bases de datos, las nubes virtuales, los newsgroup (grupos de noticias), los chat, los cuales se convierten en modalidades de interacción comunicativa que permite el flujo de información donde los miembros participan activamente, lo anterior, permite un aprendizaje permanente en el manejo de técnicas de investigación documental entre los estudiantes y docentes de las Instituciones de Educación Superior y la comunidad científica en general.

Las actividades de las universidades se direccionan a la formación de un estudiante auto gestor del conocimiento, soportado en herramientas informáticas que, con el pasar del tiempo y su permanente actualización, se han convertido en aliadas para los investigadores, alumnos y docentes, dentro de los diferentes roles para la construcción de nuevo conocimiento.

Sin embargo, hay que decir, finalmente, que es muy importante motivar a los investigadores, estudiantes y profesores, en el valor del soporte documental que se encuentra en las bibliotecas y hemerotecas, lugares en donde las visitas han disminuido notoriamente por el uso de las herramientas informáticas. 


\section{Referencias}

Alaís Grillo, A., Leguizamón Sotto, D. V., Sarmiento Ceballos, J. I. (2015). Comprensión lectora y desarrollo de estrategias cognitivas con el apoyo de un recurso TIC. Revista Educación y Desarrollo Social, 9(1), 132-143. Obtenido de https://revistas. unimilitar.edu.co/index.php/reds/article/view/556

Álvarez Cadavid, G. M., Giraldo Ramírez, M. E., Navarro Plazas, C. d. (2017). Uso de TIC en investigación cualitativa: discusión y tendencias en la literatura. KATHARSIS(23), 218-235. Obtenido de http://search.ebscohost.com/login. aspx $?$ direct $=$ true $\& \mathrm{db}=$ fap $\& A N=123097711 \&$ lang $=$ es $\&$ si te $=$ ehost-live

Angel Uribe, I. C., Vallejo Gómez, M., Zambrano Acosta, J. (2015). Las TIC en las prácticas de estudio: en la búsqueda de estrategias. Revista Q, 9(18), 1 - 20. Obtenido de http://revistaq.upb.edu.co/articulos/ver/502

Arbeláez Gómez, M. C. (2014). Las tecnologías de la información y la. Investigaciones Andina, 997-1000. Obtenido de http://www.scielo.org.co/pdf/inan/v16n29/ v16n29a01.pdf.

Arcila Calderón, C., Piñuel Raigada, J. L., Calderín Cruz, M. (2013). La e-investigación de la Comunicación: actitudes, herramientas y prácticas en investigadores iberoamericanos. Revista Comunicar, XX(40), 111-118. doi:http://dx.doi. org/10.3916/C40-2013-03-01

Arévalo Ascanio, J. G., Bayona Trillos, R. A., Rico Bautista, D. W. (2015). El problema de la brecha tecnológica: Un asunto de cultura. Sinapsis, 43-57. Obtenido de http://www.eam.edu.co/ojs/index.php/sinapsis/article/view/74/125

Becerra, L. E., Zárate Rueda, R. y Rodríguez Quiñónez, D. A. (2015). Gestión de la Investigación Universitaria: un escenario académico para la apropiación social del conocimiento. Revista Internacional de Tecnología, Conocimiento y Bienestar, 4(2), 215 - 226. Obtenido de http://search.ebscohost.com/login. aspx direct $=$ true $\& \mathrm{db}=$ fap $\& A N=111149347 \&$ lang $=$ es $\&$ si te $=$ ehost-live

Berzosa Ramos, I., Arroyo González, M. J. (2016). Docentes y TIC: un encuentro necesario. Contextos educativos (19), 147 - 159. doi:10.18172/con.2767

Capanegra, H. A., Cabrera, G., Aguilar , M. L., \& Jorda, M. S. (2016). El empleo de las tecnologías de información y comunicaciones (TICs) en el ámbito universitario. Documentos y Aportes en Administración Pública y Gestión Estatal, 16(26), 159190. Obtenido de http://www.redalyc.org/articulo.oa?id=337546668006

Caro González, F., Díez de Castro, E. (2005). Investigación cualitativa asistida por ordenador en economía de la empresa. Investigaciones Europeas de Dirección y 
Economía de la Empresa, 11(2), 45-58. Obtenido de http://www.redaedem.org/ articulos/iedee/v11/112045.pdf

Caro, A., Fuentes, A., Soto, M. (2013). Desarrollando sistemas de información centrados en la calidad de datos. Revista chilena de ingeniería, 21(1), 54-69. doi:http://dx.doi.org/10.4067/S0718-33052013000100006

Carvajal Torres, A. A., De la Torre F, N. (2016). Aprendizaje en metodología de investigación para la construcción de la escala diferencial semántico apoyado con mediaciones tecnológicas. Revista EAN, 117 - 129. Obtenido de http://journal. ean.edu.co/index.php/Revista/article/view/1461

Castillo, L. (2005). Una mirada a la academia y la investigación en ambientes virtuales: sus caracteristicas basadas en el uso de las tecnologías de la información y la comunicacion (TIC). Revista de la comunidad académica UDES, 2, 35-41. doi:http://www.oei.es/historico/n11611.htm

Cobo Romaní, J. (2009). El concepto de tecnologías de la información. Zer, 14(27), 295-318. Obtenido de http://www.ehu.eus/ojs/index.php/Zer/article/ view/2636/2184.

Colas Bravo, P., De Pablos Pons, J. (2012). Aplicaciones de las Tecnologías de la Información y la Comunicación en la Investigación Cualitativa. Revista Española de Pedagogia, 77-92. Obtenido de https://www.researchgate.net/ publication/236869834_Aplicaciones_de_las_Tecnologias_de_la_informacion_y_ la_comunicacion_n_la_investigacion_cualitativa.

Crovi Druetta, D. (2002). Sociedad de la información y el conocimiento. Entre el optimismo y la desesperanza. Revista Mexicana de Ciencias Políticas y Sociales, XLV(185), 13-32. Obtenido de http://www.redalyc.org/articulo.oa?id $=42118502$

Escofet Roig, A., López, M., Álvarez, G. (2014). Una mirada crítica sobre los nativos digitales: análisis de los usos formales de TIC entre estudiantes universitarios. Revista Q, 9(17), 1 - 18. Obtenido de http://search.ebscohost.com/login. aspx direct $=$ true $\& \mathrm{db}=\mathrm{a} 9 \mathrm{~h} \& \mathrm{AN}=117729034 \&$ lang $=$ es\&si te $=$ ehost-live

Fernández Díaz, E., Calvo Salvador, A. (2013). Estrategias para la mejora de la práctica docente. una investigacion accion colaborativa para el uso innovador de las TIC. Revista Electrónica Interuniversitaria de Formación del Profesorado, 121133. Obtenido de http://www.aufop.com/aufop/revistas/arta/digital/178/1791

Fernández Olaskoaga, L., Fernández Díaz, E., Gutiérrez Esteban, P. (2014). La colaboración docente como base para la innovación y la investigación en los entornos de aprendizaje con TIC. Revista de Docencia Universitaria, 12(4), 303 322. Obtenido de https://polipapers.upv.es/index.php/REDU/article/view/5625 
Gamboa Mora, M. C., García Sandoval, Y. (2012). Aprender haciendo en Investigación como estrategia de aprendizaje. Revista de investigaciones $U N A D(2), \quad 77$ - 93. Obtenido de http://search.ebscohost.com/login. aspx direct $=$ true $\& \mathrm{db}=$ fap $\& A N=94059360 \&$ lang $=$ es $\&$ sit $e=$ ehost-live

Gandía, C. L. (2015). Las TIC en las prácticas científicas de Investigadores: aproximaciones desde la sociología de las emociones. Sapiens Research, 5(2), 27-32. Obtenido de http://search.ebscohost.com/login. aspx ?direct $=$ true $\& \mathrm{db}=\mathrm{a} 9 \mathrm{~h} \& \mathrm{AN}=110232184 \&$ lang $=$ es\&si te $=$ ehost-live

Grané Oró, M., Crescenzi Lanna, L., Olmedo Casas, K. (2013). Cambios en el uso y la concepción de las TIC, implementando el Mobile Learning. RED(37), 1-19. Obtenido de http://search.ebscohost.com/login. aspx?direct $=$ true $\& \mathrm{db}=$ fap $\& A N=89983923 \&$ lang $=$ es\&sit $\mathrm{e}=$ ehost-live

Gros, B. (2016). Retos y tendencias sobre el futuro de la investigación acerca del aprendizaje con tecnologías digitales. Revista de Educación a Distancia RED(50), 2 - 13. doi:http://dx.doi.org/10.6018/red/50/10

Guacaneme Mahecha, M., Zambrano Izquierdo, D., Gómez Zermeño, M. G. (2016). Apropiación tecnológica de los profesores: el uso de recursos educativos abiertos. Apropiación tecnológica de los profesores: el, 105 - 117. doi:10.5294/ edu.2016.19.1.6

Gutiérrez G, M. C. (2005). La formación pedagógica investigativa en estudiantes de formación avanzada en educación. Revista de Ciencias Humanas UTP, 11(35), 173-182. Obtenido de http://revistas.utp.edu.co/index.php/chumanas/article/ view/1073/587

Heerkens, G. (2002). Gestión de Proyectos. (S. M.-H. ESPAÑA, Ed.) España: Mc.GrawHill. doi:https://www.casadellibro.com/libro-gestion-de-proyectos-defina-unproyecto-seleccione-un-equipo-m-antenga-el-control/9788448137311/860365

Henao, L. J., Cuartas Ramírez, D. (2016). Plan estratégico de TIC para el grupo de investigación en Gestión de la. Gestión de las personas y tecnología (26), 81-93. Obtenido de: https://dialnet.unirioja.es/descarga/articulo/5771044.pdf

Isaza Domínguez, L. G., Vargas Guativa, J. A., Preciado, C. M. (2016). Estrategia pedagógica para la apropiación del uso de las tecnologías de la información y la comunicación (TIC) para docentes de educación superior. Revista Virtual Universidad Católica del Norte(49), 92-109. Obtenido de http://revistavirtual.ucn. edu.co/index.php/RevistaUCN/article/view/799/1319

Jornet Melia, J., González Such, J., García Bellido, M. (2012). La Investigación Evaluativa y las Tecnologías de la Información y la Comunicación (TIC). Española 
de Pedagogia(251), 93-110. Obtenido de https://dialnet.unirioja.es/servlet/ articulo? codigo $=3803601$

Lotero Echeverri, G. J. (2016). Formación de capacidades para fomentar el diálogo entre culturas, a través de las Tic en Medellín (Colombia). Revista Lasallista de investigación, 13(1), 116 -125. Obtenido de http://search.ebscohost.com/login. aspx $?$ direct $=$ true $\& d b=a 9 h \& A N=119193315 \&$ lang $=$ es\&si te $=$ ehost-live

Martínez Flores, K., Torres Barzabal, L. M. (2017). Strategies that helps the University Teacher to know, assimilate and implement the ICT in the Classroom. Innovation Table. Píxel-Bit. Revista de Medios y Educación, 159-175. Obtenido de http://hdl. handle.net/11441/51924

Mirete Ruiz, A. B. (2016). El profesorado universitario y las TIC. Análisis de su competencia digital. Ensayos (31), 133 - 147. Obtenido de http://www.revista. uclm.es/index.php/ensayos Mruck, K. (2005). Proporcionar (en línea) recursos y servicios para investigadores cualitativos: desafíos y posibilidades. Forum Qualitative Sozialforschung / Forum: Qualitative Social Research, 6(2), 38. Obtenido de http://nbn-resolving.de/urn:nbn:de:0114-fqs0502388

Navarrete Calao, L., Vargas Arteaga, J. (2015). Comprensión de lectura y producción de discurso escrito mediados por TIC, en educación media. 3 ciencias, 4(4), 244 - 254. Obtenido de https://www.3ciencias.com/articulos/articulo/comprensionde-lectura-y-produccion-de- discurso-escrito-mediados-por-tic-en-educacionmedia/

Nieto Martin, S., Rodriguez Conde, M. J. (2007). Convergencia de resultados en dos diseños de investigación-innovación en enseñanza universitaria a través de las TIC. Revista Española de Pedagogia (236), 27 - 48. Obtenido de http://search. ebscohost.com/login. aspx ?direct $=$ true $\& d b=a 9 h \& A N=25260005 \& l a n g=e s \& s i t$ $\mathrm{e}=$ ehost-live

Olivares Carmona, K. M., Angulo Armenta, J., Torres Gastelú, C., Madrid García, E. M. (2016). Las TIC en educación: metaanálisis sobre investigación y líneas emergentes en México. Apertura, 8(2), 100 - 115. doi:http://dx.doi.org/10.18381/ Ap.v8n2.866

Pardo Gómez, M., Izquierdo-Lao, J., Sánchez Ramírez, L. (2010). El Proceso de Formación para la Investigación Científica en la Educación Superior Sustentada en las Tecnologías de la Información y las Comunicaciones. Pedagogía Universitaria, xv(2), 132-143. Obtenido de http://www.redalyc.org/articulo. oa?id $=181317848011$

Paz Saavedra, L. E., Fierro Marcillo, Y. D. (2015). Competencias investigativas en los docentes beneficiados por la estrategia de formación y acceso para la apropiación 
pedagógica de las TIC. Tendencias, XVI(1), 175 - 194. Obtenido de http://search. ebscohost.com/login.aspx? direct $=$ true $\& \mathrm{db}=$ fap $\& A N=103140856 \& l a n g=e s \& s i$ te $=$ ehost-live

Pérez Pinzón, L. R. (2014). Tecnología e informática de la historia. Resistencias e innovaciones en el uso investigativo y pedagógico de las TICs. Revista Científica Electrónica de Ciencias Humanas ORBIS(29), 67 - 93. Obtenido de http://www. redalyc.org/html/709/70932556004/

Pérez, D., Dressler, M. (2006). Tecnologías de la información para la gestión del. Intangible Capital, 15(3), 31-59. Obtenido de http://upcommons.upc.edu/ bitstream/handle/2099/2945/Tecnologias\%20de\%20la\%20informacion.pdf? sequence $=1$

Ramírez-de-La-Piscina-Martínez, T. (2013). Los lipdubs, como herramientas de conquista social. Estudio de cuatro casos paradigmáticos realizados en Quebec,EEUU, Cataluña y País Vasco. Revista Latina de Comunicación Social (68), 55-88. doi:10.4185/RLCS-2013-969.

Rubiano Arciniégas, D. A., Gómez Flórez, L. C. (2012). Comunidades de práctica soportadas en tecnologías de información como apoyo al desarrollo de las líneas estratégicas de investigación en las instituciones de educación superior. Revista del Instituto de Estudios en Educación Universidad del Norte (16), 42 - 53. Obtenido de http://rcientificas.uninorte.edu.co/index.php/zona/article/viewArticle/2342

Ruiz Bolívar, C. (2014). Evaluación de una experiencia de tutoría virtual de tesis de grado en el contexto de un programa de doctorado en educación. Paradigma, 35(1), 129-148. Obtenido de http://search.ebscohost.com/login. aspx direct $=$ true $\& \mathrm{db}=$ fap $\& A N=98273462 \&$ lang $=$ es\&sit $e=$ ehost-live

Sánchez Ramírez, L., Pardo Gómez, M. E., Izquierdo Lao, J. M. (2010). La dinámica del proceso de formación para la investigación científica en la educación superior sustentada en las tecnologías de la información y las comunicaciones. Pedagogía Universitaria, XV (2), 76 -81. Obtenido de www.redalyc.org/ pdf/1813/181317848011.pdf

Sánchez Robayo, B. J., Torres Duarte, J. (2016). Aprender a investigar investigando. realización de una propuesta de formación. Revista Científica CIDC, 63 - 87. doi:10.14483/udistrital.jour.RC.2017.28.a2

Salado Rodríguez, L. (2011). Contribución de los recursos educativos abiertos al aprendizaje significativo de las Tecnologías de Información y Comunicación en el estudiante universitario. Revista Internacional Administración \& Finanzas, 4(1), 101-114. Obtenido de http://www.theibfr.com/ARCHIVE/RIAF-V4N1-2011.pdf 
Sanz, C., Cukierman, U., Zangara, A., Santángelo, H., González, A., Rozenhauz, J., Ibañez , E. (2006). Integración de la tecnología móvil a los entornos virtuales de enseñanza y de. En II Congreso en Tecnología en Educación y Educación en Tecnología. TE\&ET'07, Universidad Nacional de la Plata, 117-126. Obtenido de http://sedici. unlp.edu.ar/bitstream/handle/10915/19120/Documento_completo.pdf?sequenc $\mathrm{e}=1$

Sobrado Fernández, L. (2006). Las competencias de los orientadores en el ámbito de las TIC (Tecnologías de la Información y la Comunicación): diagnóstico y desarrollo. Estudios Sobre Educación, 11, 27-43. Obtenido de http://dadun.unav. edu/bitstream/10171/8957/1/Eb.pdf

Tejedor Tejedor , F. J., Garcia, A., Muñoz -Repiso, V. (2012). Sociedad tecnológica e investigación educativa. Revista española de pedagogía rep (251), 3-26. Obtenido de http://search.ebscohost.com/login. aspx?direct $=$ true $\& d b=$ fap $\& A N=71491563$ \&lang $=$ es\&sit $\mathrm{e}=$ ehost-live

Torres Velandia, S. Á., Jaimes Cruz, K. (2015). Producción de conocimiento mediado por TIC: cuerpos académicos de tres universidades públicas estatales de México. Revista electronica de educación Sinéctica, 1-16. Obtenido de http://search. ebscohost.com/login.aspx?direct $=$ true $\& \mathrm{db}=\mathrm{a} 9 \mathrm{~h} \& \mathrm{AN}=109233127 \&$ lang $=$ es\&si te $=$ ehost-live

Torres Velandia, S. Á., Aguilar Tamayo, M. F., Girardo, S., Villalobos Hernández, M. M. (2012). Modelos, chacia una Sociedad del Conocimiento? consideraciones a partir del desarrollo de la ciencia, la educación superior y las TIC. Revista Electrónica de Investigación Educativa, 14(2), 34-51. Obtenido de http://redie.uabc.mx/redie/ article/view/307 